www.jmscr.igmpublication.org Impact Factor 5.244

Index Copernicus Value: 5.88 ISSN (e)-2347-176x ISSN (p) 2455-0450 crossref DOI: _http://dx.doi.org/10.18535/jmscr/v4i5.35

\title{
Hyperthyroidism Induced Dilated Cardiomyopathy is Reversible
}

\author{
Author \\ Dr Gopalakrishnan \\ MD, DCARD,FICC, Consultant Cardiologist, Dept of Cardiology, Holy Family Hospital, Kerala
}

\begin{abstract}
Dilated Cardiomyopathy is an uncommon presentation in hyperthyroid patients. There are very few case reports of Hyperthyroidism induced Dilated cardiomyopathy, which is completely reversible.
\end{abstract}

\section{INTRODUCTION}

Cardiomyopathy as an initial presentation has been reported in $6 \%$ of hyperthyroid patients. while less than $1 \%$ of them developed dilated cardiomyopathy with severe left ventricular dysfunction.

Mechanisms described below which direct action of Thyroid hormone on the cardiac muscle may cause cardiomyopathy. Hyperthyroid induced Dilated cardiomyopathy is reversible described in this case report.

\section{CASE REPORT}

A 74 year-old non-diabetic, non-hypertensive female presented with history of shortness of breath, paroxysmal nocturnal dyspnea, pedal edema. She also complained of swelling over neck of 1 year duration, loss of weight of 4 month duration, and generalized weakness of 30 days duration. She had no similar complaints in the past and had no history of coronary artery disease/Bronchial Asthma/Tuberculosis/Chronic obstructive pulmonary disease. She is a non smoker and never consumed alcohol.
On examination, she was thin built; pale, with no icterus, pedal edema. Among vital signs; Temperature was 98.6, pulse rate (PR) was 90/min, regular, blood pressure (BP) was 130/80mm Hg, respiratory rate (RR) was 16/min. Cardiovascular system examination showed jugular venous pressure (JVP) was not elevated. Cardiomegaly was present, apex in 6th left inter costal space (ICS), in anterior axillary line, forceful, S1, S2 heard normal, Ejection Systolic Murmur heard over pulmonary area, no other murmurs or rub, Respiratory System was normal, Abdomen was soft and there were no masses. Central nervous system showed generalized wasting of muscles, resting tremor, deep tendon reflexes (DTR): $3+$ bilaterally. Thyroid examination showed swelling in the neck moving with deglutition, Diffusely enlarged and no bruit heard over the swelling All other systems were normal.

Her investigations revealed as below. Among Complete blood picture (CBP), Hemoglobin was 8.5 gm\%, Red Blood Cell (RBC) count was 3.61 million/cumm, Packed Cell Volume (PCV) was 26.2, Total Platelet Count (TPC) was 1.92 
Lakh/cumm, Total leukocyte count (TLC) was 7500/ cumm, N60, L35, M3, E3, B0, ESR was 20 $\mathrm{mm}$, Blood Urea $24 \mathrm{mg}$, Serum Creatinine was $0.7 \mathrm{mg}$, Complete Urine Examination (CUE) NAD, Random Blood Sugar (RBS) was 142 mg/dl, Serum Electrolytes: Na - 135 m eEq/L, K $3.6 \mathrm{mEq} / \mathrm{L}, \mathrm{CL}-109 \mathrm{mEq} / \mathrm{L}$. ECG showed sinus Tachycardia,LAE, LVH, 2D ECHO showed Global hypokinesia of Left Ventricle with EF $31 \%$, moderate MR,mild AR / TR , Moderate $\mathrm{PAH}$, severe LV dysfunction+, no PE/Clot, Viral Markers showed HIV - negative, HbsAg negative, HCV - negative. T3 - $7.49 \mathrm{nmol} / \mathrm{l}(0.95$ - 2.5), T4 >320 nmol/l $(60-120), \mathrm{TSH}<0.01$ $\mu \mathrm{IU} / \mathrm{L}(0.25$ - 5).

The patient was treated with Diuretics, ACE inhibitors, Digoxin, Neomercazole and Nebivolol with a regular follow up. 2D-echo was repeated after 4 months show complete improvement in the ejection fraction. The thyroid profile normalized after 4 months of treatment and remained normal on subsequent follow up with treatment.

\section{DISCUSSION}

Cardiac dysfunction in hyperthyroid induced dilated cardiomyopathy is believed to be caused by changes in ventricular volumes, contractility and heart rate. It may produce circulatory changes, reduce circulation time. The increased cardiac work load leads to cardiac hypertrophy, which results in reduction in the contractile reserve that is reflected in the failure of exercise to create an increase in the ejection fraction, a pattern that can be reversed after thyroid suppression treatment.

\section{REFERENCES}

1. Nayak B, Burman K. Thyrotoxicosis and thyroid storm. Endocrinol Metab Clin North Am., 2006; 35(4): 663-686, vii.

2. Fadel B. M., Ellahham S., Lindsay J., Ringel M. D., Wartofsky L., Burman K. D. Hyperthyroid heart disease. Clinical cardiology, 2000; 23(6): 402- 408.
3. Forfar JC, Muir AL, Sawers SA, Toft AD. Abnormal left ventricular function in hyperthyroidism: evidence for a possible reversible cardiomyopathy. N Eng J Med 1982;307:1165-70.

4. Bauerlein EJ, Chakko CS, Kessler KM. Reversible dilated cardiomyopathy due to thyrotoxicosis. Am J Cardio 1992;70:1323

5. Ravindran V, Mathur DS, Gupta AK, et al. Reversible dilated cardiomyopathy associated with thyrotoxicosis. J Assoc Physicians India 2001;49:660-1. 